\title{
ANALYSIS OF PLANTS AND SEDIMENTS FROM THE TIDELANDS OF THE COASTAL REGIONS OF THE TOHOKU DISTRICT FOLLOWING THE 2011 TSUNAMI
}

\author{
K. SERA and F. BABA \\ Cyclotron Research Center, Iwate Medical University \\ 348-58 Tomegamori, Takizawa, Iwate 020-0173, Japan \\ S. GOTO, C. TAKAHASHI, and Y. SAITOH \\ Takizawa Laboratory, Japan Radioisotope Association \\ 348-58 Tomegamori, Takizawa, Iwate 020-0173, Japan \\ M. MATSUMASA \\ Department of Biology, Center for Liberal Arts and Sciences, Iwate Medical University, \\ 2-1-1 Nishitokuta, Yahaba, Iwate 028-3694, Japan
}

\begin{abstract}
The tidelands (tidal flats) of the seaside district of Tohoku, Japan, which play an important role in maintaining the diversity of the marine ecosystem, were almost completely destroyed by the massive tsunami that occurred on March 11, 2011. The tsunami also brought large quantities of contaminated sludge from the bottom of the sea to the coastal lands including the tidelands. In this study, we collected sludge samples from three tidelands: the Tsugaruishi estuary tideland (Iwate pref.), the Orikasa estuary tideland (Iwate pref.), and the Gamou tideland (Miyagi pref.). We then analyzed the samples using PIXE in accordance with a powdered-internal-standard method that we developed. In order to investigate the effects of the contaminated sludge on the ecosystem, we gathered and analyzed various kinds of wild plants and seaweed. We found that the tidelands are contaminated with heavy elements, such as arsenic, lead, zirconium, and barium, although the contamination is less serious in the vicinity of the beach.
\end{abstract}

Keywords: PIXE, heavy elements, tsunami sludges, tidelands, marine ecosystems

\section{Introduction}

Most of the tidelands are located within estuaries where sea water and fresh water mix, and these areas therefore have a diversified marine ecosystem. The Sanriku district is the primary district for oysters, short-necked clams, and scallops, and the estuaries in this area form the principal habitat for these marine organisms. The structure and ecosystem of these estuaries were nearly destroyed by the large tsunami that occurred on March 11, 2011. Regeneration of these estuaries and tidelands is essential to both the reconstruction of the fishery and fish culturing industries and also to the recovery of the marine ecosystem in the Sanriku district.

The tsunami not only completely destroyed most portions of the tidelands, but also transported a large quantity of sludges from the bottom of the sea to the land. Recently, it 
has been confirmed that sea waters near the shore of the Sanriku district are contaminated with heavy elements, such as arsenic, lead, and cadmium ${ }^{1}$ that may have originated from abandoned mines, industrial waste matters, the hot springs of the inland areas, or heavy oils emitted from fishing boats. The sludge powders form aerosols after drying that are blown upwards, and the effect of these particles on human health creates a serious problem. We have recently started to study the negative effects of sludge on human health. It has been found that the sludges deposited on the urban districts contain a large amount of heavy toxic elements spread over a wide area in the Tohoku district. These results are presented in a separate paper, also submitted for publication in the proceedings of the BioPIXE 7 symposium ${ }^{2}$. It is also anticipated that the heavy element contamination of the tidelands will become an obstacle to the regeneration of the ecosystem in the estuaries and the tidelands.

In the present study, sludge samples were collected from three tidelands: the Tsugaruishi estuary tideland (Iwate pref.), the Orikasa estuary tideland (Iwate pref.), and the Gamou tideland (Miyagi pref.). The last is a well studied tideland of the Tohoku district and is known to have a rich ecosystem that exhibits a high diversity of species and biomass. However, almost all portions of this tideland were lost to the tsunami. On a portion of the tidelands, the populations of some marine animals, such as short-necked clams, decreased sharply, although the population of seafowl returned. In this study, we also collected and analyzed wild plants (including seaweed) from the tidelands in order to evaluate the effects of heavy elements on the ecosystem.

\section{Experimental}

\subsection{Sampling}

Sampling of the sludges and plants on or near the tidelands was performed twice, on August 31 and September 3, 2011, at low tide. On August 31st, 12 sludge and 10 plant samples were gathered from the Tsugaruishi estuary tideland, and three sludge and three plant samples were collected from the Orikasa estuary tideland. Fig. 1 shows the locations of these tidelands. Although nearly half of the Tsugaruishi tideland and most portions of the Orikasa tideland were lost to the Tsunami, sampling was able to be carried out on the residual areas of these tidelands, as illustrated in Fig. 2. On September 3rd, sampling was performed on or near the Gamou tideland located very close to the Sendai harbor. Although most portions of this tideland were lost during the tsunami, the regeneration of the tideland appears to be

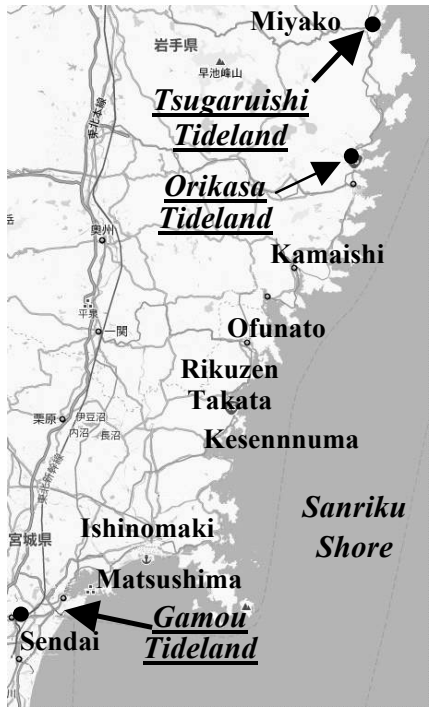

Fig. 1 Locations of the three tidelands.

relatively swift due to its high sedimentation rate. Five plant samples were taken from the small tideland near the estuary of the Nanakita river. Table 1 shows a list of plant samples collected. Additionally, ten sludge samples were collected from the remaining tidelands and from a site located one block inside the breakwater (see Fig. 6). 


\subsection{Target preparation}

The sludge samples were placed in a sterilizer and dried at $105{ }^{\circ} \mathrm{C}$ for several hours. An internal standard of palladium-carbon in the amount of $10,000 \mathrm{ppm}$ was added to the samples in accordance with the powdered-internal-standard method $^{3}$. The samples were homogenized well in an agate mortar and a few milligrams of the mixture was placed on a backing film ( $4 \mu \mathrm{m}$ thick Prolene film) and fixed with a $1 \%$ collodion solution. The plant samples were treated in accordance with the internal-standard method combined with a chemical-ashing method, where indium was added as an internal standard in the amount of $1,000 \mathrm{ppm}$, and the samples were dissolved in nitric acid in a microwave oven ${ }^{4}$.

\subsection{Irradiating and measuring conditions}

The targets were bombarded with a $2.9 \mathrm{MeV}$ proton beam extracted from a cyclotron, and the emitted X-rays were simultaneously measured Table 1 Plants collected from the three tidelands.

\begin{tabular}{|c|c|c|}
\hline & A: Hamaninniku & Elymus mollis \\
\hline & B: Hachijouna & Sonchus brachyotus \\
\hline & C: Bouaonori & \begin{tabular}{|l} 
Enteromorpha \\
intestinalis
\end{tabular} \\
\hline & D: Futojuzumo & Chaetomorpha spiralis \\
\hline & E:Aosa no issyeu & Ulva sp. \\
\hline & F: Ushinokegusa & Festuca pratensis \\
\hline & G: Hamahirugao & Calystegiasoldanella \\
\hline & H:Kauatsurigusa & Cyperus microiria \\
\hline & I: Kurara & Sophora flavescens \\
\hline & J: Hamanashi & Rosa rugosa. \\
\hline & K: Hamanigana & Ixeris repens \\
\hline & L: Yoshi & Phragmites australis \\
\hline & A: Nobudou & \begin{tabular}{|l} 
Ampelopsis glandulosa \\
var. heterophylla
\end{tabular} \\
\hline 5 & B: Otokoyomogi & $\begin{array}{l}\text { Artemisia indica var. } \\
\text { maximowiczii }\end{array}$ \\
\hline & A: Shiroza & Chenopodium album \\
\hline & B: Hakobe & Stellaria \\
\hline & C; $\mathrm{K}$ & \begin{tabular}{|l|} 
Indigofera \\
pseudotinctoria
\end{tabular} \\
\hline & $\begin{array}{l}\text { D:America } \\
\text { sendanngusa }\end{array}$ & Bidens frondosa \\
\hline & E: Suberihiyu & Portulaca oleracea \\
\hline
\end{tabular}
with two $\mathrm{Si}(\mathrm{Li})$ detectors $^{5}$. A $500 \mu \mathrm{m}$ thick Mylar film was used to absorb the X-rays for the No. 1 detector. To analyze the sludge samples, which contained a large amount of iron, a specially designed X-ray absorber ${ }^{6}$ was also used to obtain sensitive measurements of the heavy elements. In Fig. 3, the spectrum of a typical sludge sample obtained with the specially designed absorber is shown. The beam spot size, the typical beam current and the measuring time were $6 \mathrm{~mm}$ in diameter, $30 \mathrm{nA}$, and 3 to 10 minutes, respectively. When the specially designed absorber was used, the beam currents were around $100 \mathrm{nA}$.



Fig. 2 Sampling spots on Tsugaruishi (1) and Orikasa (2) tidelands, where C, D and E in Tsugaruishi tideland are seaweeds.
Fig. 3 Typical spectrum of a sludge sample (Tsugaruishi -a) obtained with a specially designed absorber developed for improving sensitivity for heavy elements. Palladium was added to the sample as an internal standard. 


\section{Results and discussion}

Fig. 4 shows the results for the soil samples collected from the three tidelands. The surface of the tidelands is colored in black, which is one of the features of contaminated sludge. However, the concentration of toxic elements is not as high as that found in the sludge samples that were deposited on the areas inhabited by people ${ }^{2}$ (shown in the black dotted line on the figure). It is possible that the heavy elements in the sludge were washed away at full tide. In addition, the concentration of toxic elements is almost the same as the average value obtained for 57 soil samples gathered from the inland of the Iwate prefecture (shown in the wide gray line). On the other hand, the concentration of heavy elements, such as $\mathrm{As}, \mathrm{Zr}$, and $\mathrm{Ba}$, measured in the samples collected near the Gamou tideland from inside a coastal levee is relatively high, as shown in Fig. 5. The locations of these sampling spots are shown in Fig. 6.

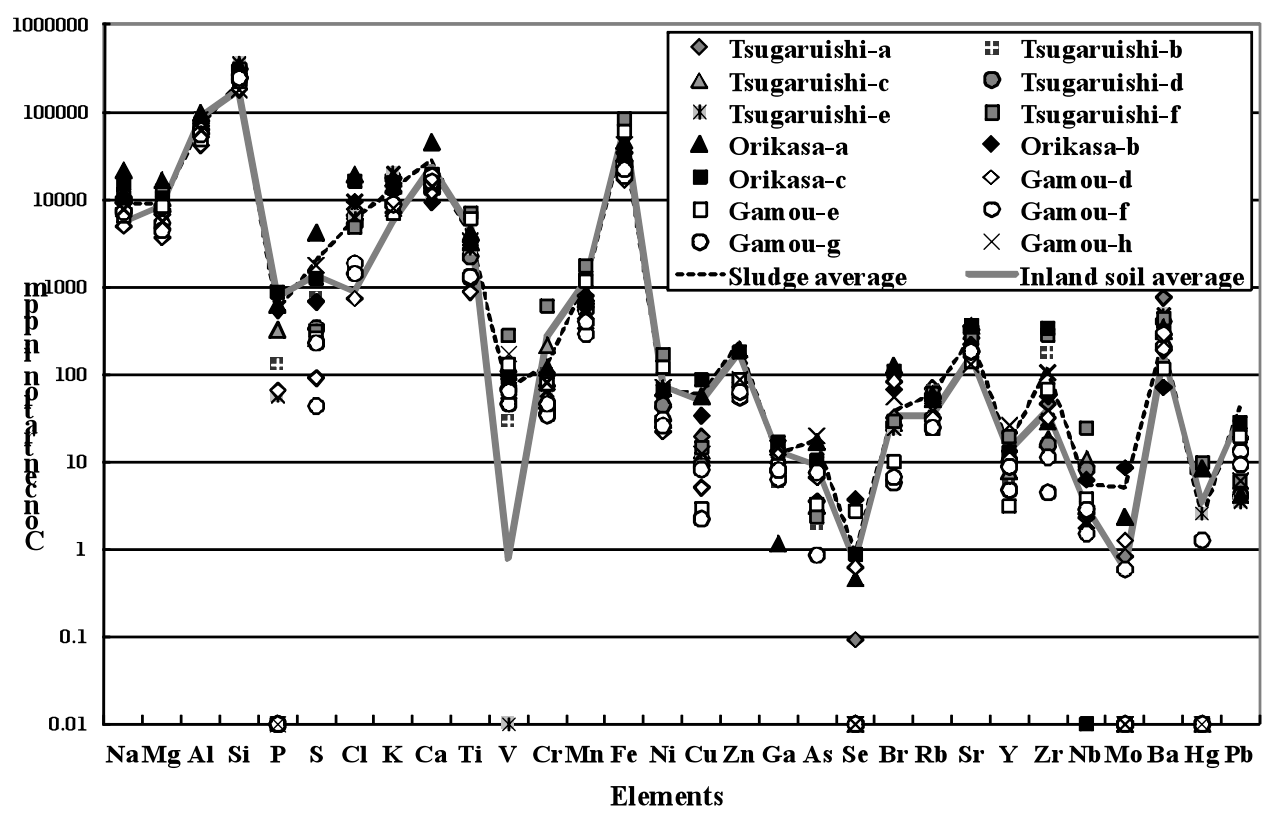

Fig. 4 Elemental concentrations of the soil samples collected from the three tidelands.

The concentration of heavy elements, such as $\mathrm{Pb}, \mathrm{Br}$, and $\mathrm{Zr}$, found in the plant samples collected from the three tidelands are higher in comparison with that found in the wild plants collected from the inland of the Tohoku district. A comparison of these values is given in Fig. 7 (Tsugaruishi) and Fig. 8 (Orikasa \& Gamou). As demonstrated in these figures, the concentrations of $\mathrm{Ca}, \mathrm{Ti}, \mathrm{Mn}$, and $\mathrm{Rb}$ was found to be higher in the plant samples obtained from the inlands, while the concentrations of $\mathrm{Zr}, \mathrm{Na}, \mathrm{Mg}, \mathrm{Si}, \mathrm{S}, \mathrm{Cl}, \mathrm{Cr}$, $\mathrm{Se}$, and $\mathrm{Pb}$ were found to be higher in the plant samples obtained from the tidelands. High concentrations of $\mathrm{Cr}$ and $\mathrm{Pb}$ might exert negative effects upon the marine ecosystem through the food chain since these elements are absorbed by phytoplankton. However, the concentration of As and $\mathrm{Hg}$ were found to be almost the same. 


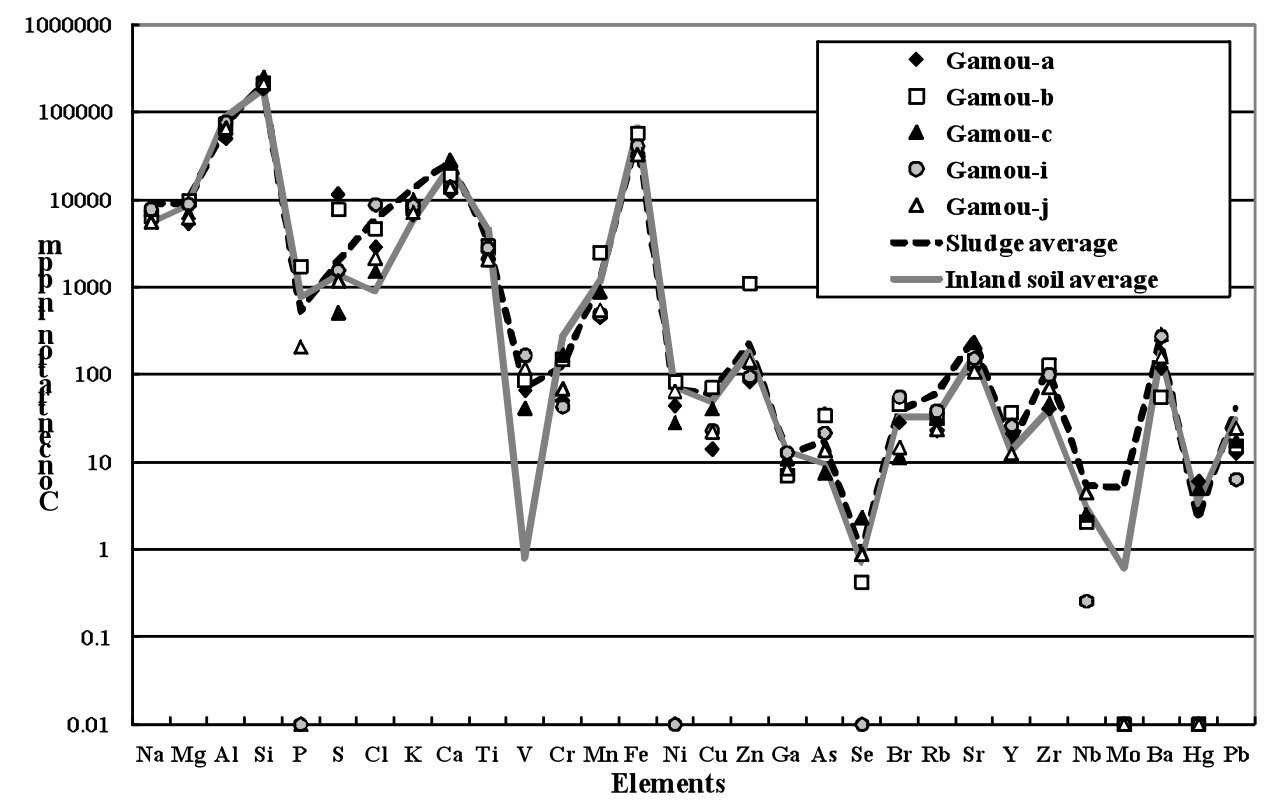

Fig. 5 Elemental concentrations of the sludge samples collected near the Gamou tideland inside a coastal levee.

The sludge samples that were collected one block inland from the breakwater on the Gamou tideland show higher concentrations of heavy toxic elements. It is expected that the sludge accumulating on the bottom of the Sendai harbor is highly contaminated; consequently, the sludge deposited on the land near the harbor is also contaminated. However, the tideland is covered with seawater at high tide, which is expected to wash away most of the heavy elements located there. On the other hand, wild plants began to grow shortly after the tsunami disaster, and these plants have already absorbed a certain amount of heavy elements. Moreover, it is known that dissolved arsenic is readily taken up by phytoplankton and by invertebrates, such as barnacles and oysters (mainly into their shells) ${ }^{7}$, and monitoring of these organisms will be needed in the future.

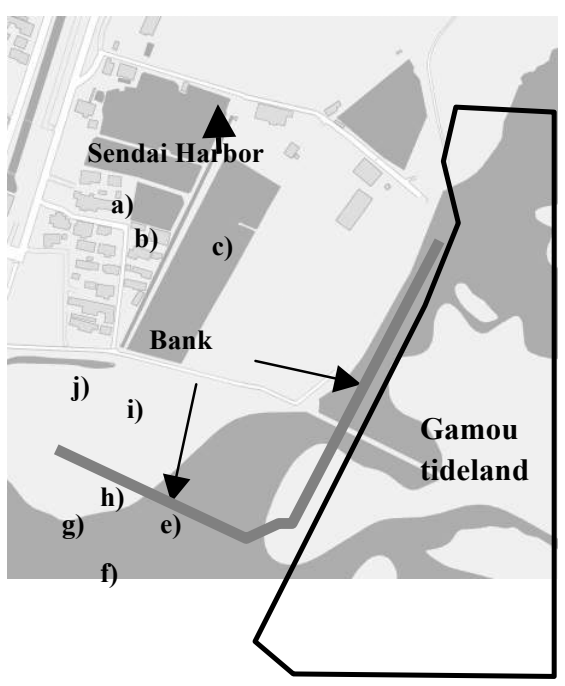

FFig. 6 Sampling spots of sludge samples on and near Gamou Tideland. 


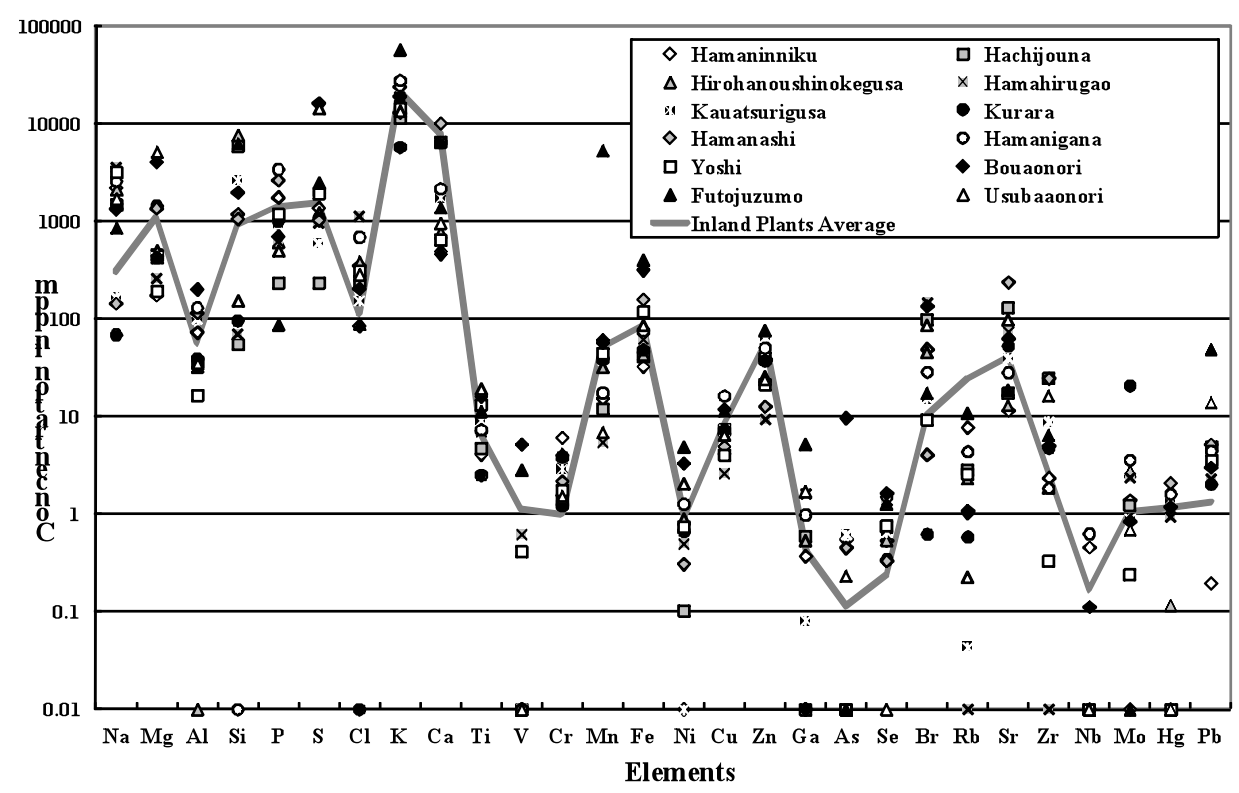

Fig. 7 Elemental concentration in 12 kinds of plants collected from the Tsugaruishi Tidelands, where average concentration of 45 wild plants collected from the inland district is shown in the solid line.

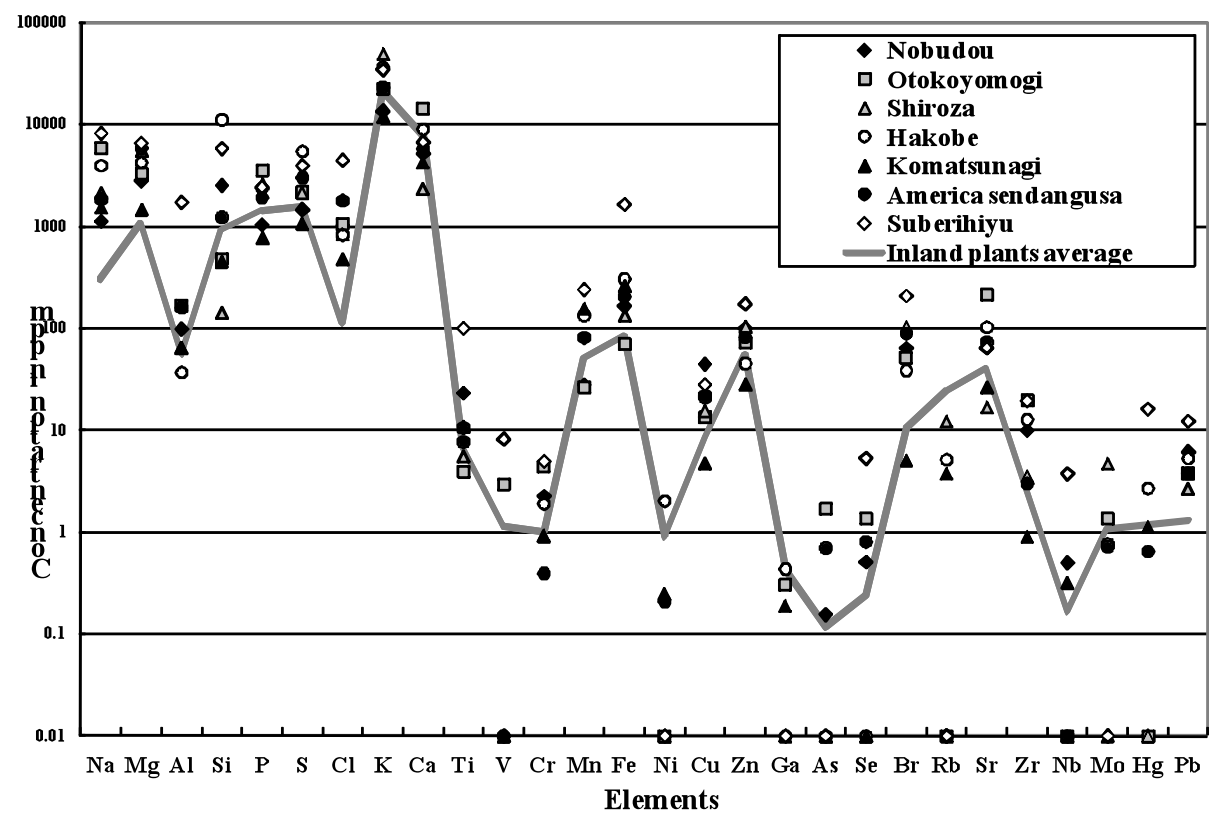

Fig. 8 Same as Fig. 7 but for seven plant samples collected from the Orikasa and the Gamou Tidelands.. 


\section{Conclusions}

The sludge deposited on the tidelands was found to be less contaminated with heavy elements than that deposited on the wharves and urban districts. The concentration of some heavy elements, such as chromium and lead, were found to be greater in plants growing on the tidelands than that found in inland plants. It is expected that monitoring the concentration of heavy elements in marine organisms will provide meaningful information regarding the recovery of the marine ecosystem

\section{Acknowledgements}

We wish to thank the staff of the Cyclotron Research Center for their help with this work. We also thank to those who helped with the sampling of the studied districts.

\section{References}

1. J. Itoh, S. Futatsugawa, Y. Saitoh, F. Ojima and K. Sera, "Application of a Powdered-internalstandard Method to Plant and Seaweed Samples.."

Int'l Journal of PIXE, Vol.15-1,2, 27-39 (2005)

2 F. Baba, K. Sera, S. Goto, C. Takahashi and Y. Saitoh, "Analysis of Contaminated Sludge Deposited on the Land Attacked by Great Tsunami following Tohoku Great Earthquake Disaster.", submitted to Int'l Journal of PIXE, (this issue)

3. K. Sera and S. Futatsugawa, "Quantitative Analysis of Powdered Samples Composed of High-Z Elements.”, Int'l Journal of PIXE, Vol. $\underline{8}-2,3,185-202$ (1998)

4. S. Futatsugawa, S. Hatakeyama, Y. Saitoh, and K. Sera, "Present Status of NMCC and Sample Preparation Method of Bio-Samples.", Int'l Journal of PIXE, Vol. $\underline{3}-4$, (1993) 319-328

5. K. Sera, T. Yanagisawa, H. Tsunoda, S. Futatsugawa, S. Hatakeyama, Y. Saitoh, S. Suzuki and H. Orihara., "Bio-PIXE at the Takizawa facility (Bio-PIXE with a baby cyclotron)", Int'l Journal of PIXE, Vol. 2, No.3 (1992) 325-330

6. K. Sera and S. Futatsugawa, "Effects of X-ray Absorbers Designed for Some Samples in PIXE Analyses. ", Int'l Journal of PIXE, Vol. 5-2,3, 181-193 (1995)

7. J. G. Sanders, R. W. Osman and G. F. Riedel, "Pathways of arsenic uptake and incorportion in estuarine phytoplankton and the filter-feeding invertebrates Eurytemora affinis, Balanus improvisus and Crassostrea virginica.", Marine Biology, Vol. 103, 3, 319-325 (1989) 